\title{
es. \\ ОПРЕДЕЛЕНИЕ РАСПРЕДЕЛЕНИЯ КОНЦЕНТРАЦИИ ВОДЯНОГО ПАРА ПО ИЗМЕРЕНИЯМ ВЕРТИКАЛЬНЫХ ЯРКОСТНЫХ ПРОФИЛЕЙ АТМОСФЕРЫ иЗ КОСМОСА
}

\author{
(Представил Г. Кузмин)
}

Проведенные в 1975 г. эксперименты с орбитальной станции «Салют-4» дали ряд вертикальных яркостных профилей атмосферы в ближней инфракрасной области спектра в разных географических районах и в различных условиях освещения Солнцем [']. Институтом астрофизики и физики атмосферы АН ЭССР и Вычислительным центром СО АН СССР разработан ряд методов решения обратной задачи определения вертикальных профилей коэффициента аэрозольного рассеяния по полученным со станции «Салют-4» интенсивностям солнечного излучения $\left[{ }^{1,2}\right]$. Настоящая работа продолжает эти исследования: приводится решение обратной задачи атмосферной оптики - определяется распределение концентрации водяного пара. Метод решения этой задачи основывается на применении методов Ньютона-Канторовича и Монте-Карло. Коэффициенты аэрозольного рассеяния рассчитывались по формуле $\left[{ }^{3}\right]$

$$
\sigma_{\lambda}(h)=f(\lambda) \sigma_{\lambda_{0}}(h),
$$

где $\lambda, \lambda_{0}$ - длины волн, $f(\lambda)$ - некоторый коэффициент, зависящий от длины волны, а $\sigma_{\lambda_{0}}(h)$ - полученное на основе алгоритмов $\left[{ }^{1,2}\right]$ значение коэффициента аэрозольного рассеяния, соответствующего длине волны $\lambda_{0}$.

\section{1. Постановка задачи}

Рассмотрим следующую модель атмосферы. На сферическую поверхность падает параллельный поток солнечного излучения. Атмосфера толщиной $H_{a}$ разбита на $n$ слоев, в каждом из которых значения коэффициентов аэрозольного $\sigma_{a}^{i}$ и молекулярного $\sigma_{M^{i}}$ рассеяния, а также концентрации водяного пара $\eta^{i}$ взяты постоянными $\quad\left(\sigma_{a}^{n+1}=\sigma_{M}^{n+1}=0\right.$, $\left.\eta^{n+1}=0\right)$.

Пусть в точке $S$ на расстоянии $H_{\mathrm{S}}$ от поверхности Земли расположен спутник, измеряющий интенсивности солнечного излучения, рассеянного атмосферой и приходящего из нее в) точку $S$ по различным направлениям.

Выберем систему координат с началом в центре Земли $O$, направив ось $z$ через перигейную точку $P$ линии визирования при $h=0$ $(h-$ расстояние от линии визирования до поверхности Земли), а 
ось $x$ таким образом, чтобы спутник лежал в $x z$-плоскости. Единичный вектор, параллельный солнечным лучам, определяется зенитным $\theta$ и азимутальным ф углами. Будем считать заданными следующие характеристики атмосферы:

a) нормированные индикатрисы молекулярного $g_{M}(\mu)$ и аэрозольного $g_{a}(h, \mu)$ рассеяния, первая из которых определяется формулой

$$
g_{M}(\mu)=3\left(1+\mu^{2}\right) / 8, \quad-1 \leqslant \mu \leqslant 1,
$$

а для задания другой атмосфера разбивается на $l$ слоев, в каждом из которых $g_{a}$ является постоянной относительно высоты

$$
g_{a}(h, \mu)=g_{a}^{i}(\mu), \quad h \in\left[h_{g}^{i}, h_{g}^{i+1}\right], \quad i=0,1, \ldots, l ;
$$

б) коэффициенты аэрозольного $\sigma_{a}^{i}$ и молекулярного $\sigma_{M^{i}}$ рассеяния;

в) альбедо земной поверхности $A$, угловой закон отражения от Земли задан «по Ламберту»;

г) интенсивности $T^{i}$ излучения солнечных лучей, рассеянных атмосферой и приходящих из нее в точку $S$ по различным направлениям $(i=1,2, \ldots, n)$.

Наблюдаемые значения интенсивностей солнечного излучения являются функциями величин $\eta^{1}, \eta^{2}, \ldots, \eta^{n}$

$$
\tau^{i}=I^{i}\left(\eta^{1}, \eta^{2}, \ldots, \eta^{n}\right),
$$

где $n-$ количество направлений наблюдения. В настоящей статье решается обратная задача определения распределения концентрации водяного пара $\eta^{i}$ по известным значениям интенсивности $T^{i}$.

\section{2. Метод решения задачи}

Поставленную в предыдущем разделе задачу будем решать итерационным методом Ньютона-Канторовича [ $\left.{ }^{3}\right]$

$$
\eta_{p+1}^{i}=\eta_{p}^{i}+\Delta \eta_{p}^{i}, \quad i=1,2, \ldots, n ; \quad p=1,2,3, \ldots,
$$

где $\Delta \eta_{p}{ }^{i}$ удовлетворяет системе

$$
\sum_{j=1}^{n} \frac{\partial I^{k}\left(\eta^{1}, \eta^{2}, \ldots, \eta^{n}\right)}{\partial \eta_{j}} \Delta \eta_{p}^{j}=\widetilde{I}^{k}-I^{k}\left(\eta_{p}^{1}, \eta_{p}^{2}, \ldots, \eta_{p}^{n}\right), \quad k=1, \ldots, n
$$

Остановку итерационного процесса будем производить в случае, если

$$
\left|I^{k}-I^{k}\left(\eta_{p}^{1}, \eta_{p}^{2}, \ldots, \eta_{p}^{n}\right)\right| \leqslant \varepsilon,
$$

где $\varepsilon-$ заданное малое положительное число.

При каждом значении параметров $\eta_{p}{ }^{1}, \eta_{p}{ }^{2}, \ldots, \eta_{p}{ }^{n}$ значение интенсивности в точке $r=\left(r_{1}, r_{2}, r_{3}\right)$ и в направлении $\omega=\left(\omega_{1}, \omega_{2}, \omega_{3}\right)$ удовлетворяет уравнению $\left[{ }^{4}\right]$

$$
\begin{gathered}
I(r, \omega)=\iint_{R^{3} \Omega} \frac{W(\mu) P\left(\eta, r, r^{\prime}\right) \sigma_{s}(r) \exp \left(-\tau\left(r, r^{\prime}\right)\right)}{\left|r-r^{\prime}\right|^{2}} \times \\
\quad \times I\left(r^{\prime}, \omega^{\prime}\right) \delta\left(\omega-\frac{r-r^{\prime}}{\left|r-r^{\prime}\right|}\right) d r^{\prime} d \omega^{\prime}+\psi(r, \omega),
\end{gathered}
$$


где

$$
\begin{gathered}
W(\mu)=\left(\sigma_{a}(r) g_{a}(r, \mu)+\sigma_{M}(r) g_{M}(\mu)\right) / 2 \pi\left(\sigma_{a}(r)+\sigma_{M}(r)\right), \\
\mu=\left(\omega^{\prime}, r-r^{\prime}\right) /\left|r-r^{\prime}\right|, \quad \sigma_{s}(r)=\sigma_{a}(r)+\sigma_{M}(r),
\end{gathered}
$$

причем $\sigma_{s}(r)=\sigma_{a}{ }^{i}+\sigma_{M^{i}}$, если точка $r$ находится в $i$-м слое атмосферы; $\tau\left(r, r^{\prime}\right)$ - оптическая длина пути в направлении $\omega=r-r^{\prime}|| r-r^{\prime} \mid$ от точки $r^{\prime}$ до точки $r$

$$
\tau\left(r, r^{\prime}\right)=\int_{0}^{\left|r-r^{\prime}\right|} \sigma\left(r^{\prime}+\omega t\right) d t
$$

а $P\left(\eta, r, r^{\prime}\right)-$ функция пропускания $\left[{ }^{2}\right]$

$$
P\left(\eta, r, r^{\prime}\right)=\exp \left(-\beta\left(\int_{0}^{\left|r-r^{\prime}\right|} v\left(r^{\prime}+\omega t\right) d t\right)^{\alpha}\right),
$$

где $v(r)$ есть эффективная концентрация водяного пара

$$
v(r)=\eta(r) p(r)^{\gamma},
$$

a $p(r)$ и $\alpha, \beta, \gamma$ - давление и параметры поглощения соответственно, $\psi(r, \omega)-$ плотность начальных столкновений.

Значение интенсивности в точке $r_{i}$ и в направлении $\omega_{i}$ рассчитывалось методом сопряженных блужданий $\left[{ }^{5}\right]$ с плотностью перехода, соответствующей чистому рассеянию, т. е. модели атмосферы без поглощающих элементов. Для устранения возникающего при этом смещения «вес» частицы $Q_{n}$ необходимо умножать на $P\left(\eta, r_{n-1}, r_{n}\right)$, где $r_{n}$ - радиус-вектор $n$-й точки столкновения. На практике алгоритм сопряженных блужданий реализуется следующим образом: для оценки величины интенсивности $I^{i}$ в точке $r_{i}{ }^{*}$ (координаты спутника) и в направлении $\omega_{i}^{*}$ (направление линии визирования со спутника) моделируются траектории частиц из точки $r_{i}{ }^{*}$ в направлении - $\omega_{i}{ }^{*}$ и суммируется по всем точкам столкновения $x_{n}=\left(r_{n}, \omega_{n}\right)$ величина

$$
u\left(x_{n}\right)=Q_{n} W\left(\mu_{n}\right) \exp \left(-\tau_{0}\right) P_{0}\left(\eta, r_{n}\right), \quad \mu_{n}=\left(e_{0}, \omega_{n}\right),
$$

где $\tau_{0}$ - оптическая длина отрезка от точки столкновения $x_{n}$ до верхней границы атмосферы в направлении $e_{0}$, обратном солнечным лучам; $P_{0}\left(\eta, r_{n}\right) \longrightarrow$ значение функции пропускания на отрезке между точкой $r_{n}$ и верхней границей атмосферы в направлении $e_{0}$, а «вес» частицы $Q_{n}$ определяется из рекуррентного соотношения

$$
Q_{n}=Q_{n-1} P\left(\eta, r_{n}, r_{n-1}\right), \quad Q_{0}=1 .
$$

Осредненная по всем траекториям случайная величина

$$
\xi=\sum_{n=1}^{m} Q_{n} \psi\left(x_{n}\right)=\sum_{n=1}^{m} u\left(x_{n}\right)
$$

дает приближенное значение интенсивности. Погрешность счета $\delta$ c заданной вероятностью $q$ удовлетворяет неравенству $\left[{ }^{6}\right]$

$$
|\delta| \leqslant x \sqrt{D \xi / N}
$$

где $D \xi$ - дисперсия случайной величины $\xi, N$ - количество разыгранных траекторий, а $\propto$ находится из соотношения $q=\sqrt{2 / \pi} \int_{0}^{x} \exp \left(-z^{\sharp} / 2\right) d z$. 
Для вычисления производной $\partial I / \partial v_{i}$ суммируется по всем точкам столкновения величина

$$
d_{i}\left(x_{n}\right)=-\alpha \beta Q_{n} \psi\left(x_{n}\right)\left(S_{n}^{i}+R_{n}^{i}\right),
$$

где $S_{n}{ }^{i}$ и $R_{n}{ }^{i}$ определяются соотношениями

$$
\begin{aligned}
& S_{n}^{i}=S_{n-1}^{i}+\left(1 / \tau^{1-\alpha}\left(\eta, r_{n-1}, r_{n}\right)\right) l_{i}, S_{0}^{i}=0, \\
& R_{n}^{i}=\left(1 / \tau^{1-\alpha}\left(\eta, r_{n-1}, r_{n}\right)\right) l_{i}^{0} .
\end{aligned}
$$

Здесь $l_{i}{ }^{0}$ и $l_{i}$ - суммарные длины отрезков, полученных при пересечении луча $r_{n}+s e_{0}(s>0)$ и вектора $r_{n}-r_{n-1}$ соответственно с $i$-м слоем атмосферы,

$$
\begin{aligned}
& \tau\left(\eta, r_{n-1}, r_{n}\right)=\sum_{j} v_{j} l_{j}, \\
& \tau_{0}\left(\eta, r_{n}\right)=\sum_{j} v_{j} l_{j}^{0} .
\end{aligned}
$$

Формула (3) получена по схеме $\left[{ }^{7}\right]$. При этом учитывалось, что $v(r)=$ $=\eta_{i} p_{i}{ }^{\gamma}$, если $r$ находится в $i$-м слое. Отсюда

$$
P\left(\eta, r_{n-1}, r_{n}\right)=\exp \left(-\beta\left(\sum_{j} v_{j} l_{j}\right)^{\alpha}\right) \text {. }
$$

\section{3. Численные результаты}

В каждой точке столкновения «снималась» следующая информация.

ИНФ1. Значение интенсивности однократного рассеяния для модели чистого рассеяния без учета рассеяния фотонов на пути от Солнца до точки столкновения.

ИНФ2. Значение интенсивности однократного рассеяния для модели чистого рассеяния.

ИНФ3. Значение интенсивности многократного рассеяния для модели чистого рассеяния.

ИНФ4. Значение интенсивности однократного рассеяния с учетом поглощения и рассеяния солнечного излучения только от точки столкновения до спутника.

ИНФ5. Значение интенсивности однократного рассеяния с учетом поглощения водяным паром.

ИНФб. Значенйе интенсивности многократного рассеяния с учетом поглощения водяным паром (т. е. значение функции $\left.I^{i}\left(\eta^{1}, \eta^{2}, \ldots, \eta^{n}\right)\right)$.

В таблице приведены результаты счета. Индикатриса рассеяния взята из $\left[{ }^{8}\right]$, коэффициент аэрозольного рассеяния - из $\left[{ }^{1}\right]$, молекулярного - из $\left[{ }^{9}\right]$, параметры поглощения для функции пропускания из $\left[{ }^{10}\right]$. Результаты измеренных интенсивностей $\widetilde{I}^{i}$ были получены с орбитальной станции «Салют-4». Атмосфера толщиной 40 км была разбита на 8 слоев по $5 \kappa$ каждый. Альбедо земной поверхности $A=$ $=0,270 ;$ зенитный угол Солнца $\theta=72^{\circ}$; азимутальный угол $\varphi=43^{\circ}$. Расчеты проводились для длины волны $\lambda=1,9$ мки.

Для определения концентрации водяного пара потребовалось 8 итераций при довольно хорошем начальном приближснии. На одну итерацию при 2800 разыгранных траекториях потребовалось 10 мин машин- 


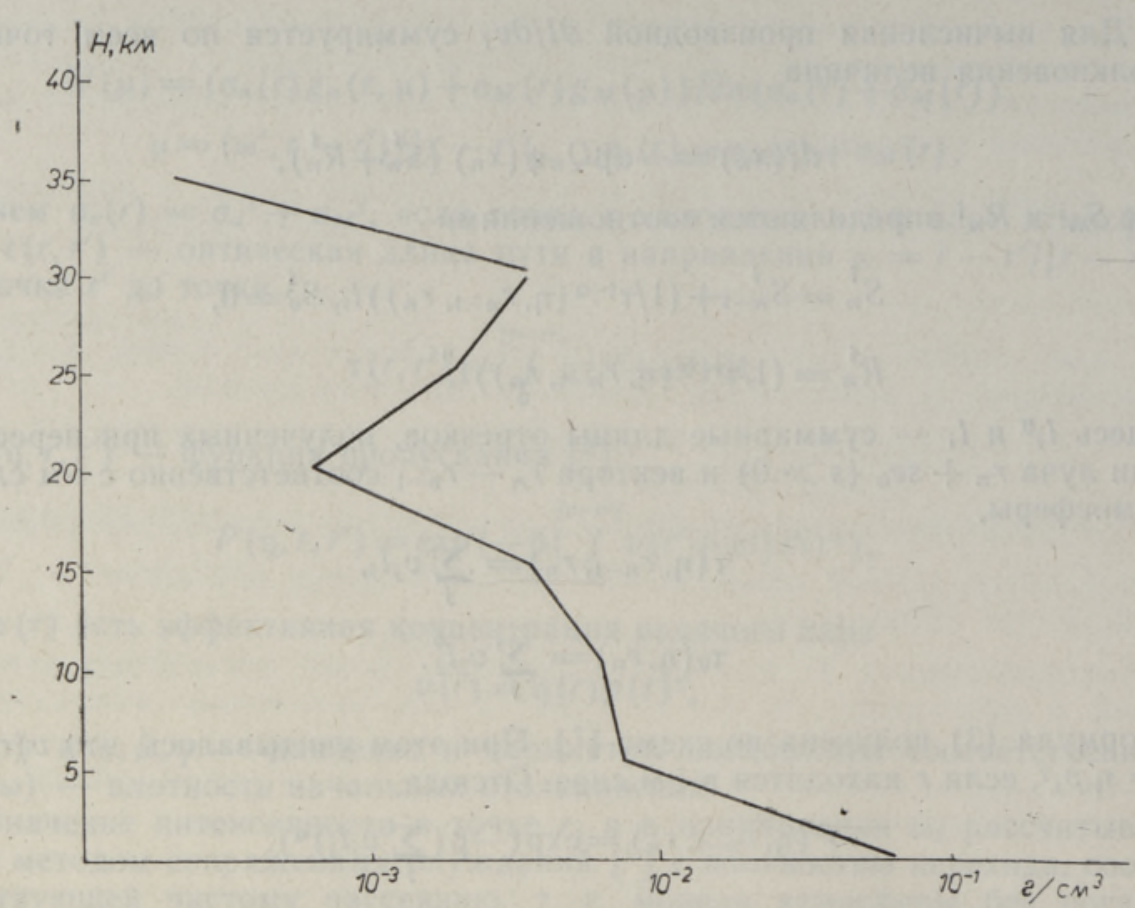

Высотный ход концентрации водяного пара.

ного времени БЭСМ-6. При этом погрешность расчета $I^{i}\left(\eta^{1}, \eta^{2}, \ldots, \eta^{n}\right)$ составляла $5 \%$ для нижних и менее $1 \%$ для верхних слоев атмосферы. На рисунке показан высотный ход полученного распределения водяного пара.

Заметим, нто параметры атмосферы и значения интенсивностей для нижних слоев были заданы с малой точностью. Поэтому значения распределения водяного пара для нижних слоев (примерно до 15 км) могут существенно отличаться от реальных величин. Суммарное количество водяного пара в интервале между $h_{1}$ и $h_{2}$ есть

$$
W\left(h_{1}, h_{2}\right)=\int_{h_{1}}^{h_{2}} \eta(h) d h ; \quad W(0,40)=0,735 c M ; \quad W(10,40)=0,121 c M
$$

\begin{tabular}{|c|c|c|c|c|c|c|c|c|}
\hline $\begin{array}{c}\text { Номер } \\
\text { слоя }\end{array}$ & 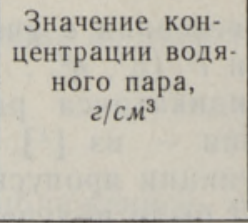 & $\begin{array}{c}\text { Высота } \\
\text { линии } \\
\text { визиро- } \\
\text { вания } \\
\text { со спу- } \\
\text { тника, } \\
\kappa м\end{array}$ & $\begin{array}{l}\text { Изме- } \\
\text { ренные } \\
\text { интен- } \\
\text { сивно- } \\
\text { сти, отн. } \\
\quad \text { ед. }\end{array}$ & ИНФ 1 & ИНФ2 & ИНФЗ & ИНФ5 & ИНФ6 \\
\hline 1 & 0,118 & 0,34 & 0,465 & 4,244 & 4,131 & 4,831 & 0,458 & 0,465 \\
\hline 2 & $0,476 \cdot 10^{-2}$ & 5,1 & 0,524 & 3,995 & 3,905 & 4,447 & 0,517 & 0.524 \\
\hline 3 & $0,122 \cdot 10^{-1}$ & 10,20 & 0,6 & 3,476 & 3,431 & 3,837 & 0,614 & 0,623 \\
\hline 4 & $0,354 \cdot 10^{-2}$ & 15,30 & 1,0 & 3,025 & 3,002 & 3,341 & 0,986 & 1,000 \\
\hline 5 & $0,835 \cdot 10^{-3}$ & 20,06 & 0,632 & 1,153 & 1,151 & 1,284 & 0,623 & 0,632 \\
\hline 6 & $0,295 \cdot 10^{-2}$ & 25,16 & 0,242 & 0,429 & 0,429 & 0,470 & 0,239 & 0,242 \\
\hline 7 & $0,452 \cdot 10^{-2}$ & 30,26 & 0,110 & 0,166 & 0,166 & 0,182 & 0,109 & 0,110 \\
\hline 8 & $0,216 \cdot 10^{-3}$ & 35,020 & 0,065 & 0,069 & 0,069 & 0,075 & 0,065 & 0,065 \\
\hline
\end{tabular}


Расчеты показывают, что вклад однократного рассеяния очень велик (таблица, ИНФ5, ИНФ6). Поэтому представляется целесообразным для моделей атмосферы с малой оптической толщиной развивать методы типа

$$
I_{1}^{i}\left(\eta^{1}, \eta^{2}, \ldots, \eta^{n}\right)=T^{i}, \quad i=1,2, \ldots, n,
$$

где $I_{1}{ }^{i}-$ значение интенсивности однократного рассеяния.

Сопоставляя результаты ИНФ1 и ИНФ2, можно видеть, что для моделей чистого рассеяния с малыми зенитными углами Солнца ослаблением лучей от Солнца до точки столкновения при расчетах однократного рассеяния можно пренебречь. Это облегчает составление алгоритмов для решения обратных задач по определению коэффициентов аэрозольного рассеяния. Заметим, что анализ ИНФІ, ИНФ2 и ИНФ3 подтверждает правомерность использования алгоритмов типа $\left[{ }^{1}\right]$ для решения обратных задач по определению коэффициентов аэрозольного рассеяния для атмосферы чистого рассеяния.

Однако анализ ИНФ4 и ИНФ5 показывает, что ослабление излучения на пути от Солнца до точки столкновения существенно влияет на значение интенсивности однократного рассеяния для модели с поглощающими элементами.

Автор выражает благодарность сотрудникам отдела методов МонтеКарло ВЦ СО АН СССР В. С. Антюфееву и М. А. Назаралиеву за практическую помощь при составлении и реализации алгоритмов МонтеКарло.

\section{ЛИТЕРАТ У РА}

1. Авасте О. А., Вей сманн У. К., Виллманн Ч. И. и др., В кн.: Исследования атмосферно-оптических явлений с борта орбитальной научной станции «Салют-4», Тарту, изд. АН ЭССР, 1979, с. 158-166.

2. Ав асте О. А., Антюфеев В. С., В айникко Г. М. и др., Там же, c. $146-157$.

3. Кан торович Л. В., Акилов Г. П., Функциональный анализ, М., «Наука», 1979.

4. Михайлов Г. А., Некоторые вопросы теории методов Монте-Қарло, Новосибирск, «Наука», 1974.

5. М ар чук Г. И., Мих айлов Г. А., Н аз а рали ев М. А., Д ар бинян Р. А., К а ргин Б. А., Еле пов Б. С., Метод Монте-Карло в атмосферной оптике, Новосибирск, «Наука», 1976.

6. Е рм аков С. М., Метод Монте-Карло и смежные вопросы, М., «Наука», 1971.

7. Ми х айлов Г. А., Ж. вычисл. матем. и матем. физ., 7, № 4, 915-919 (1967).

8. Федорова Е. О., Решетникова Н. С., Нефедова О. В., Изв. АН СССР, Физ. атмосф. и океана, 9, № 8, 886-889 (1973).

9. McClatchey, R. A., Fenn, R. W., Selby, I. E. A., Volz, F. E., Gar ing, I. S., Optical Properties of the Atmosphere (Third Edition), Environmental Research Press, № 411, 1972.

10. Гол убицкий В. М., Москаленко Н. И., Изв. АН СССР, Физ. атмосф. и океана, 4, № 3, 346-359 (1968).

Ннститут астрофизики и физики атмосферы Академии наук Эстонской ССР
Поступила в редакцию $27 / \mathrm{XI} 1980$ 


\section{J. KNJAZIHHIN}

\section{VEEAURU KONTSENTRATSIOONI MAARAMINE ATMOSFÄARI VERTIKAALSETEST HELENDUSPROFIILIDEST (KOSMOSEST MOODETUNA)}

On esitatud veeauru kontsentratsiooni määramise tulemused kosmiliste optiliste mõōtmiste põhjal. Probleemi lahend pōhineb Newton-Kantorovitši ja Monte Carlo meetoditel.

\section{J. KNJAZIHHIN}

\section{THE DETERMINATION OF THE WATER VAPOUR CONCENTRATION FROM THE SPACE MEASUREMENTS OF THE VERTICAL BRIGHTNESS PROFILES OF THE ATMOSPHERE}

Experiments carried out aboard the orbital station "Salyut-4" in 1975 gave a number of profiles of the brightness of the atmosphere in the near-infrared region over different geographical areas for different heights of the Sun. Some methods of solving the inversion of vertical brightness profiles for the aerosol scattering coefficient were worked out $\left[{ }^{1,2}\right]$ by both the Institute of Astrophysics and Atmosperic Physics and the Computer Centre of the Siberian Branch of the USSR Academy of Sciences. The present paper is a sequel to those investigations: the inversion of the space measurements of the vertical brightness profiles of the atmosphere for the water vapour concentration is presented. The method for solving this problem is based on the Newton-Kantorovich and Monte-Carlo algorithms. The values of the aerosol scattering coefficient $\sigma_{\lambda}(h)$ have been calculated by formula $\left[{ }^{3}\right]$

$$
\sigma_{\lambda}(h)=f(\lambda) \sigma_{\lambda_{0}}(h),
$$

where $\lambda$ and $\lambda_{0}$ are wavelengths, $f(\lambda)$ is the wavelength dependent coefficient and $\sigma_{\lambda_{0}}(h)$ is the value of the aerosol scattering coefficient calculated by using the above method $\left[{ }^{1,2}\right]$ which corresponds to the wavelength $\lambda_{0}$. 